\title{
Enhanced spectral sensitivity of fibre long period gratings to refractive index of aqueous solutions utilising copper patterned coatings
}

\author{
T. Allsop ${ }^{\mathrm{a}}$, H. Dobb ${ }^{\mathrm{a}}$, R. Neal ${ }^{\mathrm{b}}$, K. Kalli ${ }^{\mathrm{c}}$, C. Themistos ${ }^{\mathrm{d}}$, B.M.A. Rahman ${ }^{\mathrm{d}}$, D.J. Webb ${ }^{\mathrm{a}}$, D. Mapps ${ }^{\mathrm{b}}$, \\ I. Bennion ${ }^{\mathrm{a}}$ \\ ${ }^{a}$ Photonics Research Group, Aston University, Aston Triangle, Birmingham, B4 7ET, UK. \\ ${ }^{b}$ Dept of Communications and Electrical Engineering, Facility of Technology, University of \\ Plymouth, Plymouth, PL4 8AA, U.K. \\ ${ }^{c}$ Higher Technical Institute, C. Kavafi Str., Aglantzia, P.O. Box 20423, 2152 Nicosia, Cyprus. \\ ${ }^{\mathrm{d}}$ Electrical, Electronic and Information Engineering Department, City University, Northampton \\ Square, London, EC1V0HB, UK.
}

\begin{abstract}
A set of long period grating devices have been fabricated in photosensitive single mode fibre coated with a series of copper rings (period of $380 \mu \mathrm{m}, 50 \%$ duty cycle and length of $4 \mathrm{~cm}$ ). The long period gratings were inscribed with a uniform UV-laser exposure across the entire length of the copper ring patterned coating. The devices ranged in copper thickness from $0.5 \mu \mathrm{m}$ to $1.5 \mu \mathrm{m}$. In addition, a control long period grating was fabricated in the same type of fibre with the same period for comparison. The refractive index and temperature spectral sensitivity of these devices were investigated and it was found that the index and temperature sensitivity is a function of the thickness of the copper rings, as supported by theoretical modelling. Furthermore, the index sensitivity of these devices in the 1.333 index region is greater than the control long period grating. The patterned $0.5 \mu \mathrm{m}$ coated long period grating gave a sensitivity of $\Delta \lambda / \Delta \mathrm{n}$ $=-74 \mathrm{~nm}$ leading to a resolution of $1.4 \times 10^{-3}$ compared to the control which had a sensitivity of $\Delta \lambda / \Delta \mathrm{n}=-32 \mathrm{~nm}$ with a resolution of $3.2 \times 10^{-3}$ in the index region of 1.320 to 1.380 (aqueous solution regime). This demonstrates a two fold increase in the sensitivity. This novel fibre long period grating device shows potential for increasing the resolution of measurements of the index of aqueous solutions.
\end{abstract}

Keywords: Index Sensing, Long Period Gratings, Coatings.

\section{INTRODUCTION}

A Long Period Grating (LPG) is an axially periodic refractive index variation inscribed in the core of a photosensitive single-mode optical fibre by ultra-violet radiation or other means, which couples light from the core to cladding modes at discrete wavelengths $[1,2]$. The study of LPGs has yielded many potential uses in the area of telecommunications and numerous sensing applications. This is because the mean wavelengths of the attenuation bands are influenced by strain, temperature, curvature and the refractive index of the surrounding medium [1-9]. In particular there has been strong interest in using long period gratings as chemical sensors [6,7,9], for example in the detection of organic aromatic compounds in paraffin [7]. Whilst the index sensitivity of most LPG based devices is greatest for solutions that have an index of approximately 1.44 , which is good for various organic hydrocarbon compounds, there is a need to increase the sensitivity of these devices in aqueous solutions and for biochemical applications, where index values are typically from 1.333 to 1.380 ; for example the effective index of a cell is $\sim 1.36$ to $1.38[10,11]$.

We report a twofold enhancement of index sensitivity of an LPG in the index regime of aqueous solutions by deposition of a patterned copper-ring structure. It was found that the index and temperature sensitivities of these LPGs are a function of the thickness of the copper rings. 


\section{FABRICATION}

A series of copper rings $(0.1 \mu \mathrm{m}$ to $1.5 \mu \mathrm{m}$ in thickness) were deposited on the surface of photosensitive fibres, using vacuum deposition combined with a carefully machined mask (period $380 \mu \mathrm{m}$, duty cycle $50 \%$ and length of $4 \mathrm{~cm}$ ) that was located between the fibre and the crucible (copper source). Long period gratings were subsequently inscribed by scanning the UV laser beam repeatedly across the entire length of the copper ring patterned coating; the fabrication evolution for multiple scans being shown in figure 1. The copper therefore acts as a mask, the UV laser producing a long period grating where there are gaps in the metal layers.

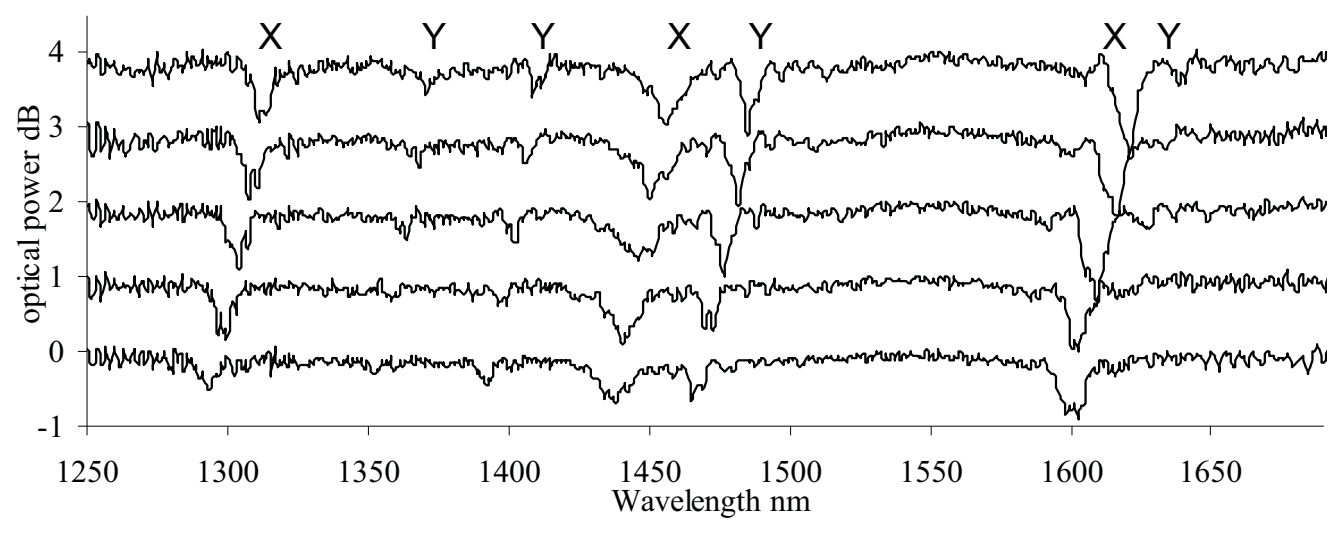

Figure 1. The evolution of transmission spectra of the copper patterned LPG (length $4 \mathrm{~cm}$, period $380 \mu \mathrm{m}$, copper thickness $0.5 \mu \mathrm{m}$ ) during fabrication using UV inscription (traces offset vertically for clarity). Bands labelled X correspond to those from the uncoated fibre (see figure 2). Bands labelled $\mathrm{Y}$ arise as a result of the coating.

On closer inspection of figure 1, it can seen that there are actually two superimposed series of attenuation bands, suggesting that the single LPG that is written interacts with two sets of cladding modes, one associated with the bare fibre waveguide and the other associated with the copper coated sections. This is the focus of our paper. Increasing the thickness of the copper coating structure red shifts the attenuation bands, with an observed shift of $44 \mathrm{~nm}$ for the attenuation band associated with the $\mathrm{HE}_{1,7}$ cladding mode with a coating thickness of $1 \mu \mathrm{m}$, see to figures $2 \mathrm{a}$ and figure $2 b$
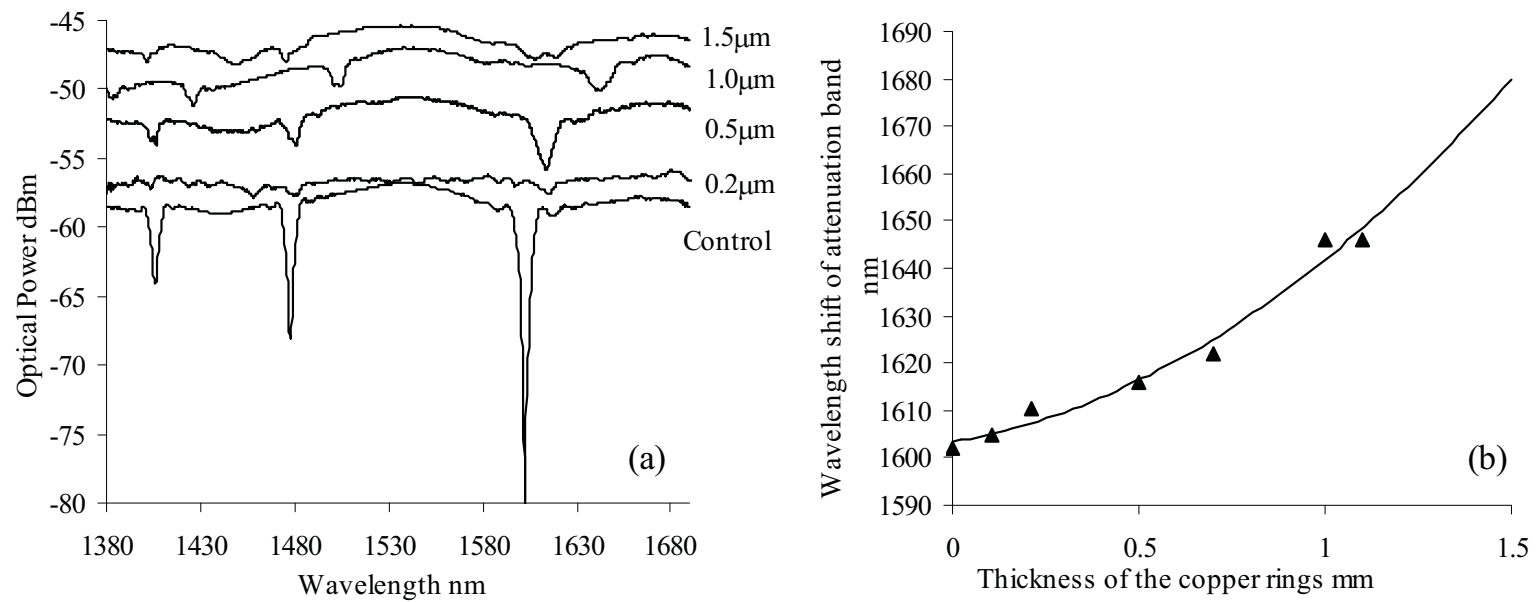

Figure 2 (a) Examples of transmission spectra for various thicknesses of copper rings. (b) The spectral wavelength shift of the attenuation band associated with the $\mathrm{HE}_{1,7}$ cladding mode as a function copper thickness. 


\section{MODELLING OF THE LONG PERIOD GRATING DEVICE}

The LPG couples incident light to the forward-propagating cladding modes of the optical fibre, which decay through radiation. The fibre supports many cladding modes, each potentially corresponding to an attenuation band in the LPG transmission spectrum. The centre wavelength $\lambda_{v}$ of an attenuation band is specified by the phase-matching condition

$$
\delta \mathrm{n}_{\mathrm{eff}} v^{\Lambda}=\lambda,
$$

where

$$
\delta n_{e f f_{v}}=\left[n_{c o}^{01}\left(\lambda, \varepsilon, T, n_{1}, n_{2}\right)-n_{c l}^{1 v}\left(\lambda, \varepsilon, n_{1}, n_{2}, n_{s,}, T\right)\right]
$$

$n_{c o} 01$ is the effective index of the core mode and $n_{c l}{ }^{1 v}$ the effective index of the $v^{\text {th }}$ radial cladding mode, both indices being dependent on the core refractive index $n_{l}$, the cladding refractive index $n_{2}$ and the wavelength $\lambda$. Moreover, $n_{c l}{ }^{1 v}$ is a function of the refractive index $n_{s}$ of the surrounding medium. $\Lambda$ is the period of the LPG, $T$ the temperature and $\varepsilon$ the strain experienced by the fibre. The quantity $\delta n_{\text {eff }}$ is the differential effective index between the core and cladding modes. In Eqn. (2), the superscripts denote the $\mathrm{LP}_{01}$ core mode and the $\mathrm{HE}_{1 v}$ axially symmetric cladding modes (from this point forward, we replace $1 v$ with $v$ ). We are assuming here that the grating consists of a circularly symmetric index perturbation that is transverse to the axis of the fibre, so that the only non-zero coupling coefficients between the core mode and the cladding modes involve cladding modes of azimuthal order $1[1,8]$. The properties of individual cladding modes may be observed through their associated attenuation bands. The two equations 1 and 2 are used to give the local cladding modes' phase-matching conditions.

The effective indices of both the core mode and the cladding modes are calculated using finite element analysis using custom coded software. In the finite element method the problem domain is suitably divided into a patchwork of subregions called 'elements'. Each element can have a different shape and size and by using many elements a complex problem can be accurately represented. A wide range of optical devices can be modelled as each element can also have different optical parameters such as anisotropic refractive index and nonlinearity, in addition to loss or gain factors. The waveguide under investigation was assumed uniform in the propagating $z$ direction and arbitrary in the cross-sectional $x$ $y$ plane. We used the penalty function method to eliminate non-physical (spurious) modes, with the complete formulation written as [12]:

$$
\omega^{2}=\frac{\left.\int(\nabla \times H)^{*} \varepsilon^{-1} \cdot(\nabla \times H) d \Omega+\left(\frac{\alpha}{\varepsilon_{0}}\right) \int(\nabla \times H)^{*}(\nabla \cdot H) \cdot d \Omega\right)}{\int H^{*} \mu \cdot \hat{H} \cdot d \Omega},
$$

where $\alpha$ is the dimensionless penalty parameter. In the vector $\mathbf{H}$-field formulation, the $\mathrm{H}_{\mathrm{x}}, \mathrm{H}_{\mathrm{y}}$ and $\mathrm{H}_{\mathrm{z}}$ field components are continuous functions over the whole transverse plane. Applying stationeries of the variational form reduces the problem to a standard eigenvalue matrix equation

$$
\mathbf{A} x-\omega^{2} \mathbf{B} x=0
$$

where $\mathbf{A}$ is a complex Hermitian matrix and $\mathbf{B}$ is a real symmetric matrix, and the eigenvectors $x$ represent the unknown field components at the nodal points for different modes with $\omega^{2}$ as their corresponding eigenvalue.

In this approach to investigate the fibre device, the $\mathrm{H}_{\mathrm{y}}$ field modes have been examined for a standard silica fibre with copper metal clad around the circumference of the fibre, using the vector H-field formulation of the finite element method [12]. The radius of the core, the cladding and the coating were considered to be $5,62.5$ and $63 \mu \mathrm{m}$, respectively The dielectric constants used for the core, the cladding and the copper were $2.093809,2.085136$ and -106.752 , 
respectively, at an operating wavelength of $1550 \mathrm{~nm}$. It should be noted that two-fold symmetry has been used for better accuracy and the quarter fibre region has been discretised into 17910 first order triangular elements. Using this finite element analysis two waveguides were modelled; firstly a bare step index profile single mode fibre with the dimensions given above and assuming a germanium/boron doped core (note that material dispersion has been considered in this approach but the values of the Sellmeir coefficients for the doped $\mathrm{Ge} / \mathrm{B}$ core are estimated) and a silica cladding. From the model constructed the effective indices of the core and cladding modes were calculated, along with their group refractive indices which are used to calculate the index sensitivity of the attenuation bands. Secondly, a model was constructed with the same specification fibre but with various thicknesses of copper coatings (thickness varied from $0.5 \mu \mathrm{m}$ to $4.0 \mu \mathrm{m}$ ); again the effective indices of the core and cladding modes were calculated along with the group indices.

Inspecting the transmission spectra of the patterned coated LPG fibre devices in figure 1, it can be seen that there appears to be two superimposed LPG transmission spectra. As a first approximation for this fibre device, it was considered as two superimposed waveguides; one consisting of a bare fibre, the other having a uniform copper coating. Using the calculated values of effective indices of core and cladding modes for both waveguides $\Lambda-\lambda$ curves were constructed: see figure 3 .

Inspecting figure 3 shows that there is a difference between the two waveguides and that there is a greater difference in phase matching condition for the $\mathrm{HE}_{1,1}$ modes compared to the $\mathrm{HE}_{1,5}$ modes. This is seen in the transmission spectra shown in figure 1 . Also it can be seen that there are significant differences in the slopes of the phase-matching curves for the cladding modes of the two waveguides, which relate to the group indices of the cladding modes. This is a significant factor in the spectral sensitivity of the attenuation bands to changes in the surrounding medium's refractive index and is investigated in a later section.

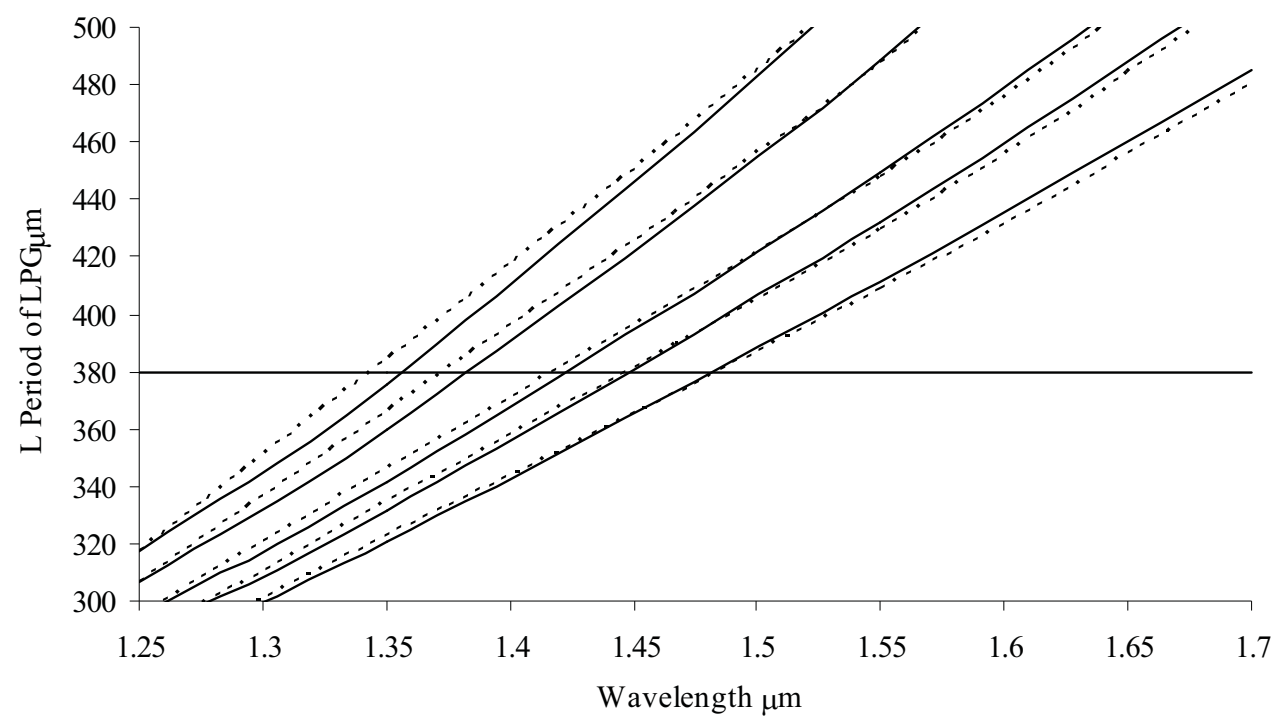

Figure 3. Examples of the finite element analysis tool calculated $\Lambda-\lambda$ curves for a ring patterned copper coated fibre LPG device (length $4 \mathrm{~cm}$, period $380 \mu \mathrm{m}$, copper, thickness $0.5 \mu \mathrm{m}$ ), showing the phase matching conditions for the attenuation bands associated with $\mathrm{HE}_{1,1}$ to $\mathrm{HE}_{1,5}$ cladding modes for the bare fibre ( - - - ) and for a $0.5 \mu \mathrm{m}$ copper coated fibre $(-)$.

\section{REFRACTIVE INDEX SENSITIVITY OF THE COPPER RINGED LONG PERIOD GRATINGS}

For the refractive index sensitivity measurements, the LPG device was placed in a V-groove and immersed in certified refractive index (CRI) liquids (supplied by Cargille laboratories Inc.) which have a quoted accuracy of \pm 0.0002 . The LPG device and V-groove were carefully cleaned, washed in methanol, then in deionised water and finally dried before the immersion of the LPG device into a CRI liquid. The V-groove was machined into an aluminium plate, to minimise 
bending of the fibre. The plate was placed on an optical table, which acted as a heat sink to maintain a constant temperature. Figures $4 \mathrm{a}$ and $5 \mathrm{a}$ show the transmission spectra as the surrounding index is increased for the coated and uncoated LPGs, respectively, Figures $4 \mathrm{~b}$ and $5 \mathrm{~b}$ show likewise the wavelength shift of the band close to $1600 \mathrm{~nm}$.
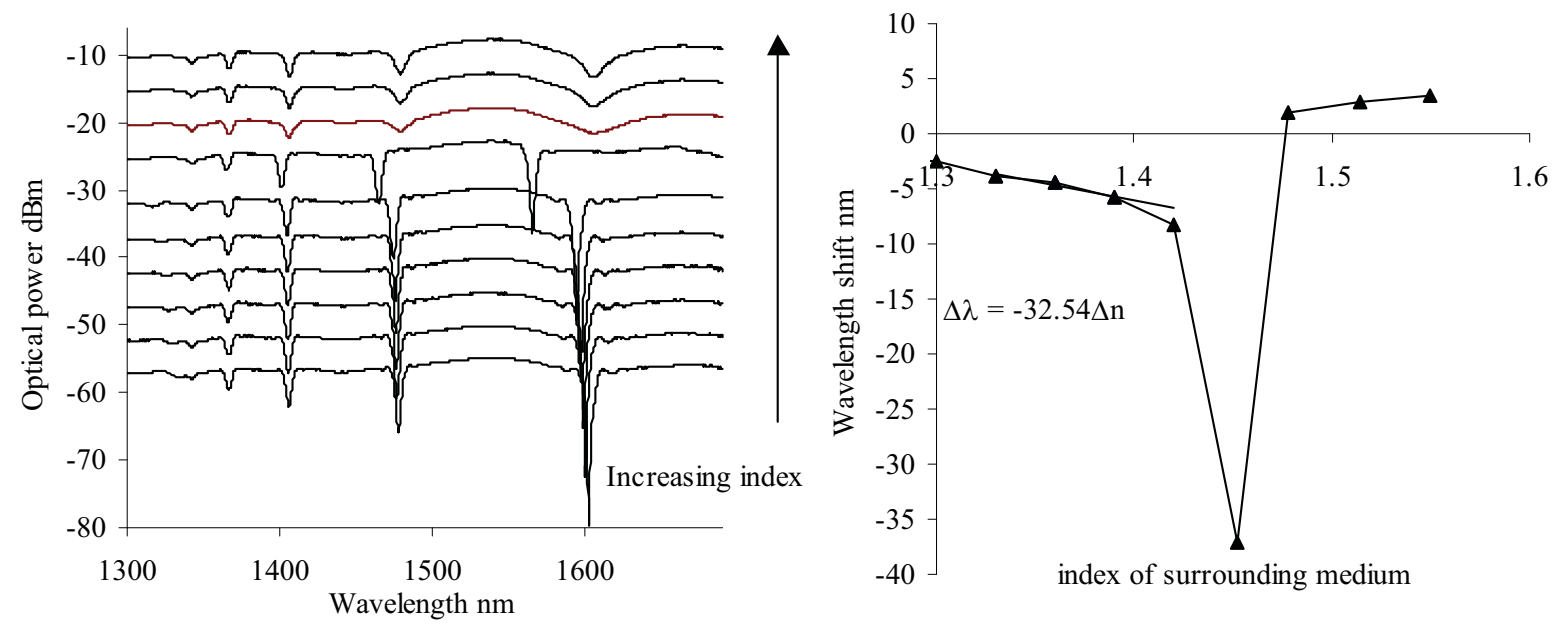

Figure 4 (a) Variation of transmission spectra of the control LPG as the surrounding medium's refractive index increases to 1.56. (b) The wavelength shift of the $1600 \mathrm{~nm}$ band as a function of the surrounding medium's refractive index.
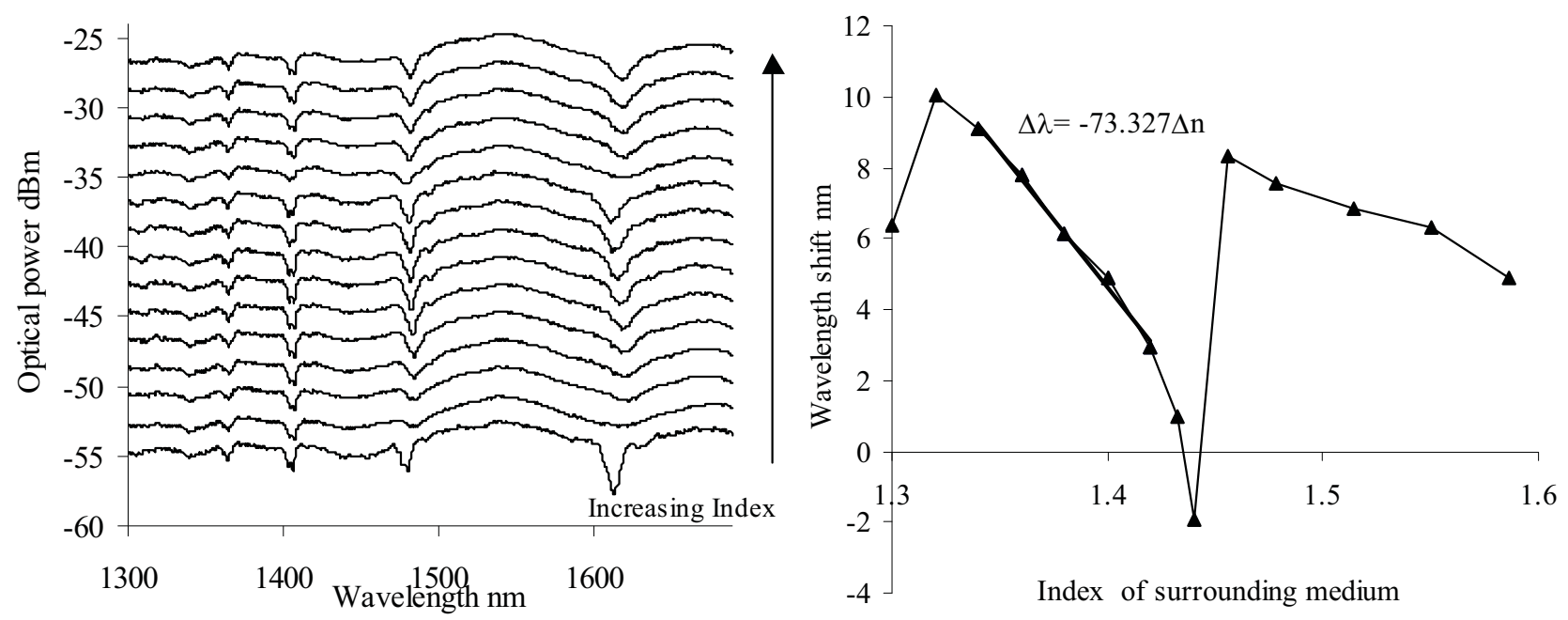

Figure 5(a) Variation of transmission spectra of the LPG coated with copper with a thickness of $0.5 \mu \mathrm{m}$ as the surrounding medium's refractive index increases to 1.56 . (b) The wavelength shift of the $1600 \mathrm{~nm}$ band as a function of the surrounding medium's refractive index.

Observing the index sensitivity of the various thicknesses of the copper patterned LPGs shows different behaviour for various index regimes. For biochemistry/biogolical applications, where the index regime of interest is from about 1.33 to 1.37 , it was found that a copper ring thickness of $0.5 \mu \mathrm{m}$ gave a maximum index sensitivity of $\delta \lambda / \delta \mathrm{n} \sim-73 \mathrm{~nm}$, as compared to the $-32 \mathrm{~nm}$ for the control LPG; a two fold increase in sensitivity. Similar experimental results for index sensitivity were found with $0.514 \mu \mathrm{m}$ and $0.714 \mu \mathrm{m}$ copper thicknesses. With coatings both thinner and thicker than this, the sensitivity decreased, see figure 6 . 


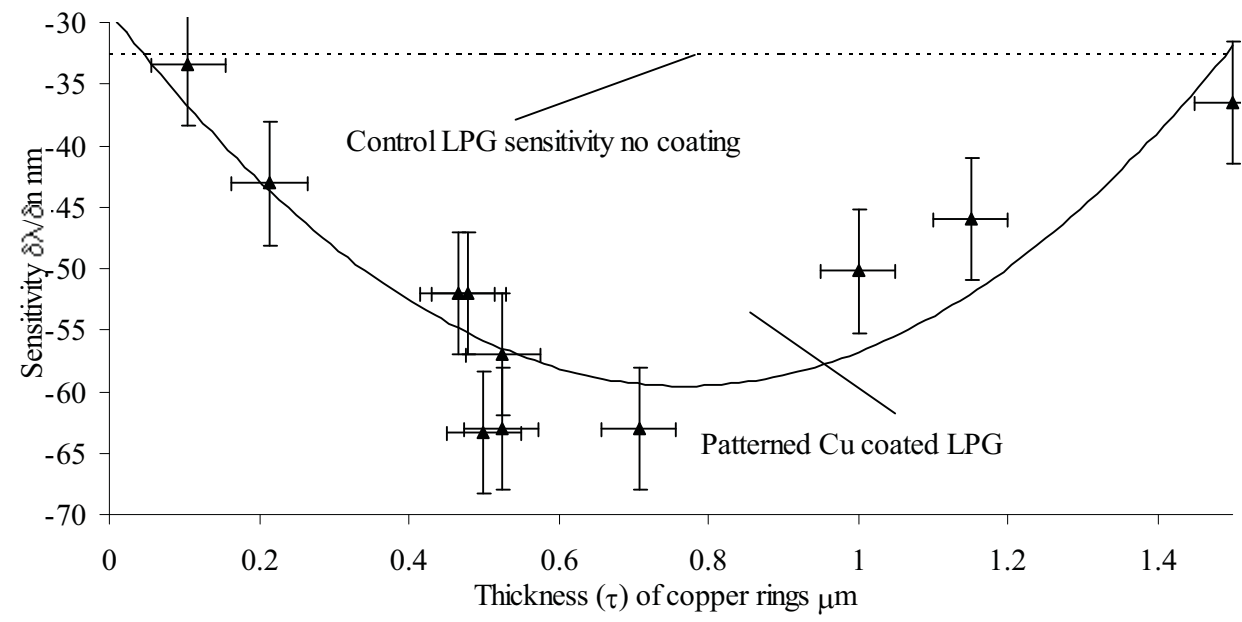

Figure 6. Measured spectral sensitivities of the various copper patterned LPGs with respect to an index regime from 1.32 to 1.38 as a function of the thickness of the copper patterns used for the LPG.

The maximum measured index sensitivity for these coated LPGs, which occurred for indices around 1.43, varied from approximately $\delta \lambda / \delta \mathrm{n}-50 \mathrm{~nm}$ to $-300 \mathrm{~nm}$, which was less than that of the control LPG: $-400 \mathrm{~nm}$. The maximum sensitivities as a function of thickness seems to be more complicated; having a decreasing cyclic sensitivity with increasing thickness of copper, see figure 7.

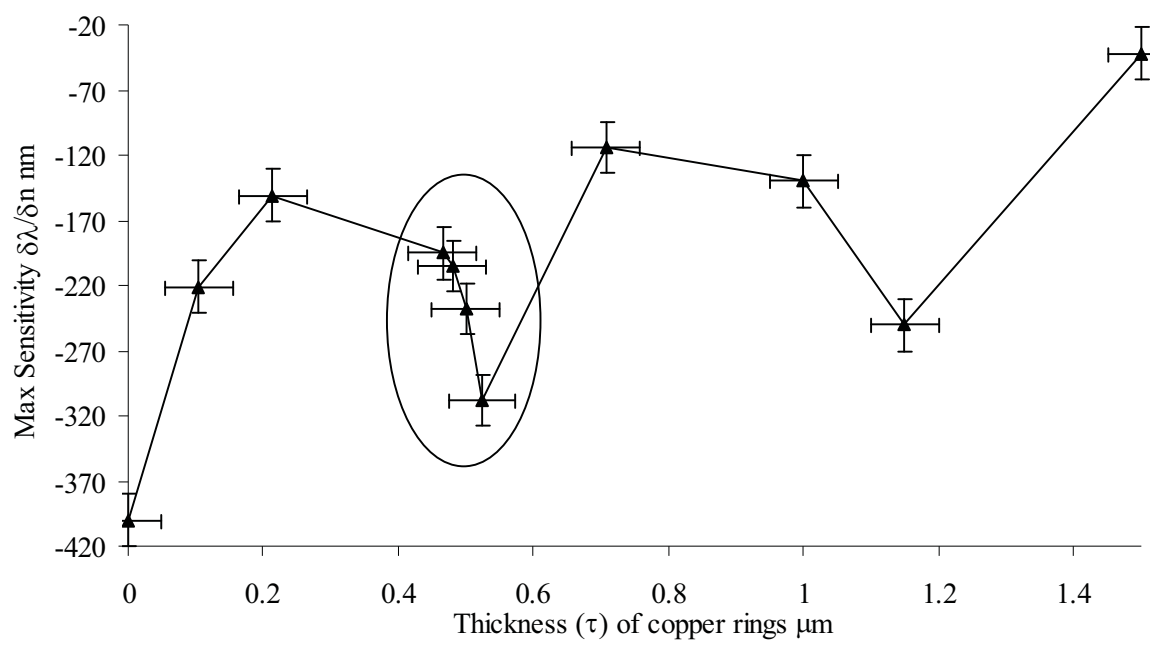

Figure 7. The maximum measured spectral sensitivities of the various copper patterned LPGs for indices from 1.42 to 1.44 as a function of the thickness of the copper patterns used for the LPG.

Inspecting all of the responses of the copper patterned LPGs it was found that varying the thickness of the copper around $0.5 \mu \mathrm{m}$ changed the spectral characteristics of the LPG devices dramatically. This is shown in figure 5 for indices of the medium around 1.3 where there is a red wavelength shift of about $+6 \mathrm{~nm}$ for $0.5 \mu \mathrm{m}$ thickness compared to $-2.2 \mathrm{~nm}$ for a thickness of $0.524 \mu \mathrm{m}$. This spectral behaviour may be exploited for measuring low indices of refraction. It has been shown from the modelling that there are effectively two superimposed waveguides and that the LPG inscribed couples to both guides; this is seen in the transmission spectra of the patterned LPG fibre device (see figure 1). Moreover, the copper thickness affects both the cladding modes' effective refractive indices and their group indices, which in turn changes the spectral sensitivity of the fibre LPG device as is seen in figures 3, 4, 5, and 6 . 
Modelling of a metal-clad square silica waveguide shows that the effective indices of those modes which exist in the metal waveguide are dependent upon the thickness of the metal, see figure 8 .

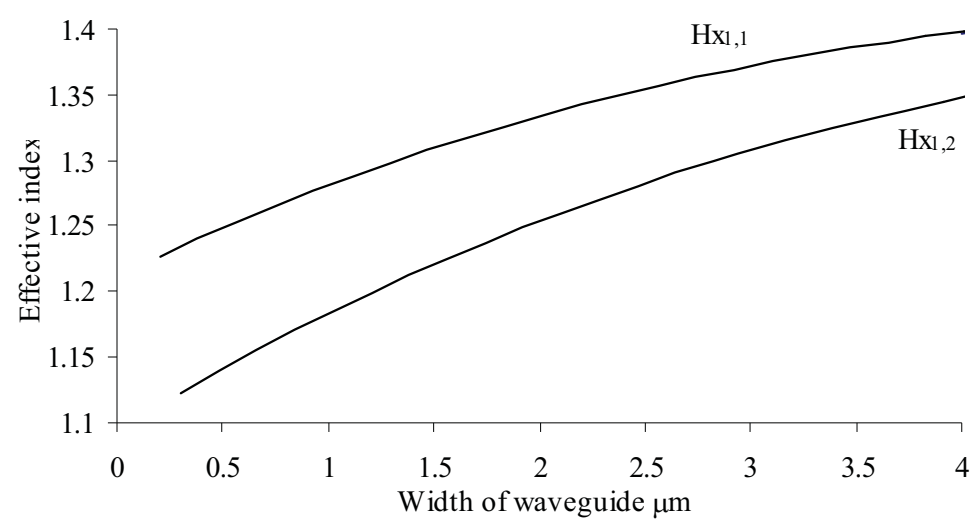

Figure 8. The theoretical $\mathrm{H}_{1,1}$ and $\mathrm{H}_{1,2}$ modes' effective indices as a function of the width of a metal clad square waveguide calculated by the use of a finite element model.

From the modelling of the copper coated LPG, we have shown that, compared to the bare fibre, the group indices of the cladding modes associated with the attenuation bands changes and this affects the sensitivity of the modes at a given wavelength. This effect will change the spectral characteristics of each LPG device with a different metal thickness; examples of this are shown in figures 4 and 5. The twofold increased sensitivity of these LPG devices for the index regime of 1.32 to 1.38 (at a thickness of $\sim 0.7 \mu \mathrm{m}$ ) occurs as metal superimposed LPGs have cladding modes with a lower effective index compared with the bare fibre LPG, hence the maximum sensitivity of the attenuation band is achieved at a lower effective index of the surrounding medium. The spectral index sensitivity of this fibre device was calculated using the approach of references [2] and [7], where it was found that the "general sensitivity parameter" for the copper coated waveguide is greater than the bare waveguide for a given cladding mode order, but the spectral index sensitivity for both waveguides were approximately the same, see figures 9 and 10. Whilst the calculated index sensitivity does not explain the experimentally observed sensitivity, the differences in the "general sensitivity parameters" may offer an indication of the spectral behaviour of the copper-coated LPG device. The results of our modelling of this fibre device reveals that a more sophisticated approach is needed.

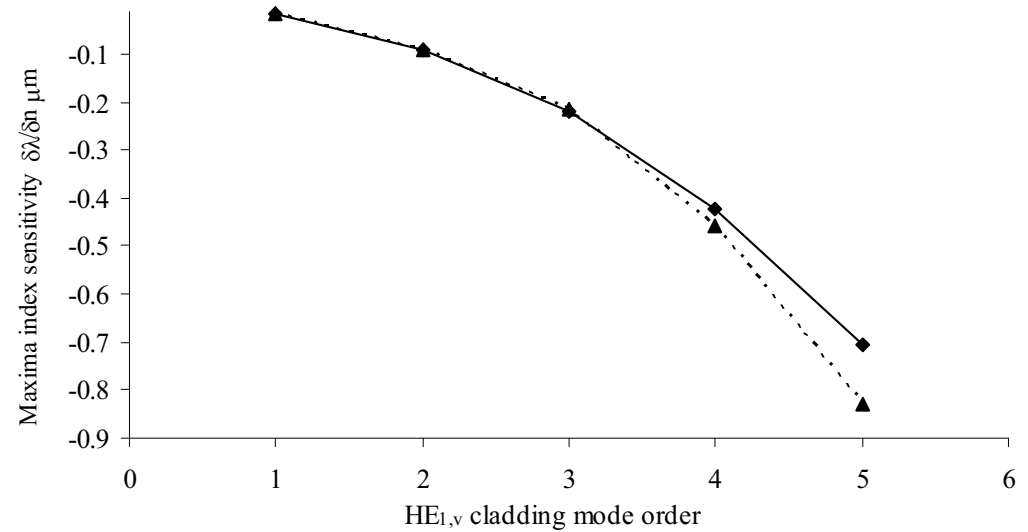

Figure 9. Calculated spectral index sensitivity for the attenuation bands associated with cladding modes $\mathrm{HE}_{1,1}$ to $\mathrm{HE}_{1,5}$ for bare (solid) and coated fibre (dashed). 


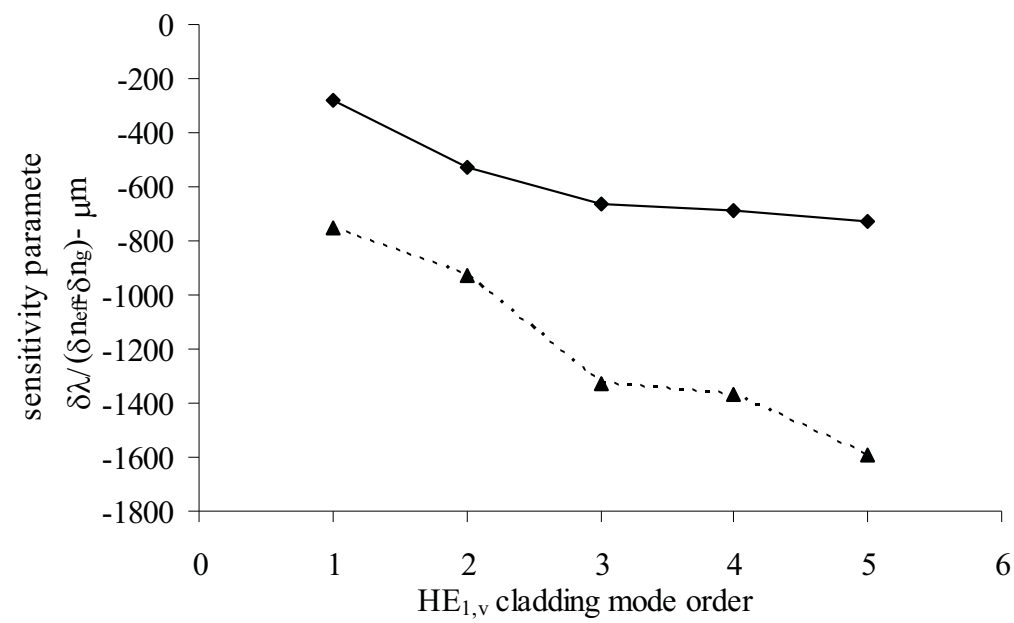

Figure 10. Calculated general sensitivity parameter for the attenuation bands associated with cladding modes $\mathrm{HE}_{1,1}$ to $\mathrm{HE}_{1,5}$ for bare (solid) and coated fibre (dashed).

\section{TEMPERATURE SENSITIVITY OF THE COPPER RINGED LONG PERIOD GRATINGS}

The experimental apparatus for measuring the temperature sensitivity of the LPGs is similar to the one for $n_{s}$, but with the LPG placed on the top of an insulated Peltier cooler. The temperature was varied and the wavelength shift of the attenuation bands of each LPG measured using an optical spectrum analyser. It was found that the thickness of the copper affected the temperature spectral sensitivity of these copper LPG devices, see figure 11.

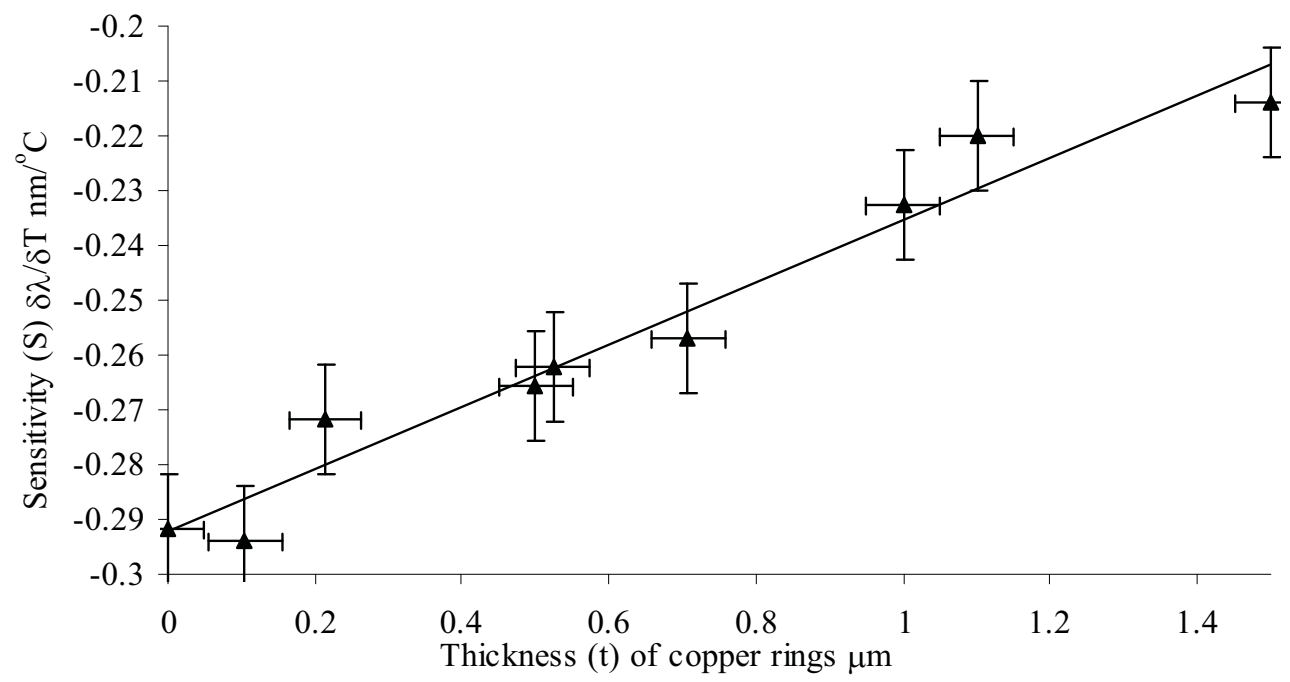

Figure 11. Temperature spectral sensitivities of the copper patterned LPG as a function of the thickness of the copper.

By increasing the thickness, the temperature sensitivity decreases from the control LPG with a temperature coefficient of $\sim-0.29 \mathrm{~nm}{ }^{\circ} \mathrm{C}^{-1}$ to $\sim-0.21 \mathrm{~nm}^{\circ} \mathrm{C}^{-1}$, which is a $27 \%$ reduction in the sensitivity from the control LPG. This temperature sensitivity dependence upon thickness of the copper appears to be a linear function with a coefficient of $0.057 \mathrm{~nm}{ }^{\circ} \mathrm{C}^{-1}$ $\mu \mathrm{m}^{-1}$. Two possible mechanisms are being investigated at the present time. The first relates to the effective and group indices of the cladding modes and how the thickness of the copper changes the cladding modes' propagation constants. 
The second concerns the thermal expansion coefficients of the glass and the copper, which are counteracting the thermooptic coefficients of the core and cladding material thus leading to an overall decrease in the spectral temperature sensitivity of these devices. The results of this investigation will be presented at the conference.

\section{CONCLUSION}

It has been experimentally demonstrated that an LPG constructed using a patterned copper coated fibre has a twofold increased sensitivity compared with a standard (bare fibre) LPG inscribed in the same fibre type, over the index range of $\sim 1.32$ to $\sim 1.38$. With a modest wavelength measurement resolution of $0.1 \mathrm{~nm}$ this implies an index resolution of $1.4 \times 10^{-3}$, which can be compared to a resolution of $3.2 \times 10^{-3}$ for the uncoated fibre. This index sensitivity is a function of the thickness of the copper pattern. These devices have been modelled using finite element analysis, and it was shown that there is an increase in general spectral sensitivity to index changes. There are differences between the calculated results and the experimental results and a more sophisticated approach is needed to take into account the whole waveguide structure of the LPG. Finally, the temperature sensitivity of these devices is shown to decrease with coating thickness, thereby reducing cross-talk between index and temperature measurements and consequently reducing the overall error in the index measurement.

\section{REFERENCES}

$1 \mathrm{~S}$. Vasilev et al, "Long-period refractive index fiber gratings: properties, applications and fabrication techniques", Proc. SPIE 4083, pp212-223, 2000

2 T. Allsop et al," A comparison and characteristics of sensing parameters of long period gratings written in three different types of fibre”, Optical Fiber Technol. No. 9, pp.210-223, 2003.

3 V. Bhatia, "Applications of long-period gratings to single and multi-parameter sensing", Opt. Express, vol. 4, no. 11, pp.457-466, 1999.

4 T. Erdogan, "Cladding-mode resonances in short- and long-period fibre Grating filters", J.Opt.Soc.Am. A, 1997, vol. 4, no.8, pp1760-1773.

5 W. Du, et al, "Long-period fibre grating bending sensors in laminated composite structures," in Sensory Phenomena and Measurement Instrumentation for Smart structures and materials. Proc. SPIE 3330, pp.284-292, 1998.

6 H.J. Patrick, "Analysis of the response of long period fiber gratings to external index of refraction", J. Lightwave Technol., Vol 16, No9, pp1606, 1998 .

7 T. Allsop et al, " Detection of Organic Aromatic compounds in Paraffin by a long period fibre grating optical sensor with optimised sensitivity", Opt. Commun. ,vol 191, pp181,2001.

8 T. Erdogan, "Cladding-mode resonances in short- and long-period fibre Grating filters", J.Opt.Soc.Am. A, 1997, vol. 4, no.8, pp1760-1773.

9 T. Allsop, et al, "High sensitivity refractometer based upon a long period grating Mach-Zehnder interferometer", Rev. Sci. Instr, Vol.73, No.4, pp.1702-1705, 2002.

10 A. Dunn and R. Richards-Kortum, “Three-dimensional computation of light scattering from cells,” IEEE J. Sel. Top. Quantum Electron. 2, pp. 898-905, 1996.

11 Joseph M. Schmitt and Gitesh Kumar, “Optical scattering properties of soft tissue: a discrete particle model”, Appl. Optics, Vol. 37, No. 13, pp.2788-2798, 1998

12 B M A Rahman and J B Davies, 'Finite-element solution of optical waveguides,' J. Lightwave Technol., 2, pp.682-687, 1984 